\title{
Fluid intake and nutritional risk in non-critically ill patients at hospital referral
}

\author{
Emanuele Cereda ${ }^{1}$, Carlo Pedrolli ${ }^{2}$, Lucio Lucchin ${ }^{3}$, Amleto D’Amicis ${ }^{4}$, Maria Gabriella Gentile ${ }^{5}$, \\ Nino Carlo Battistini ${ }^{6}$, Maria Antonia Fusco ${ }^{7}$, Augusta Palmo ${ }^{8}$ and Maurizio Muscaritoli ${ }^{9}$ on behalf \\ of the PIMAI group \\ ${ }^{1}$ Dipartimento di Scienze e Tecnologie Alimentari e Microbiologiche (DISTAM) - International Center for the Assessment \\ of Nutritional Status (ICANS), Università degli Studi di Milano, via Botticelli 21, 20133 Milano, Italy \\ ${ }^{2}$ Dietetic and Clinical Nutrition Unit, Trento Hospital, Trento, Italy \\ ${ }^{3}$ Dietetic and Clinical Nutrition Unit, Regional General Hospital, Bolzano, Italy \\ ${ }^{4}$ National Institute for Research on Food and Nutrition (INRAN), Roma, Italy \\ ${ }^{5}$ Dietetic and Clinical Nutrition Unit, 'Niguarda-Ca Granda' Hospital, Milano, Italy \\ ${ }^{6}$ Department of Applied Dietetic Technical Sciences, University of Modena and Reggio Emilia, Modena, Italy \\ ${ }^{7}$ Dietetic and Clinical Nutrition Unit, 'S.Camillo-Forlanini' Hospital, Roma, Italy \\ ${ }^{8}$ Dietetic and Clinical Nutrition Unit, University Hospital San Giovanni Battista, Torino, Italy \\ ${ }^{9}$ Department of Clinical Medicine, University 'La Sapienza', Roma, Italy
}

(Received 27 October 2009 - Revised 15 February 2010 - Accepted 16 February 2010 - First published online 7 May 2010)

\begin{abstract}
The association between hyporexia/anorexia, reduced food intake and disease-related malnutrition at hospital admission is well established. However, information on fluid intake according to nutritional risk has never been provided. Thus, we assessed the attitude and adequacy of fluid intake among case-mix hospitalised patients according to nutritional risk. A sample of 559 non-critically ill patients randomly taken from medical and surgical wards was evaluated. Nutritional risk was diagnosed by the Nutritional Risk Screening 2002. Usual fluid consumption the week before admission was assessed and categorised as $<5$ and $\geq 5 \mathrm{cups} / \mathrm{d}(1 \mathrm{cup}=240 \mathrm{ml})$, with the acceptable intake being $\geq 5 \mathrm{cups} / \mathrm{d}$. Prevalence of nutritional risk was $57.2 \%$, and $46.2 \%$ of the patients reported a fluid intake $<5$ cups/d. Multiple-adjusted logistic regression revealed that age $\geq 65$ years (OR: 1.88 (95\% CI: $1.03,3.43$ ); $P<0.04$ ), energy intake (for every $25 \%$ increase in food intake compared with estimated requirements, OR: 0.37 (95\% CI: $0.25,0.55) ; P<0.001)$ and the number of drugs taken (every three-drug increase, OR: 0.63 (95\% CI: $0.44,0.90) ; P<0.02)$ were independently associated with inadequate fluid intake $(<5$ cups/d). A significant independent association was also found with nutritional risk (OR: $0.64(95 \%$ CI: $0.43,0.95) ; P<0.03)$. Nutritional risk appears to be positively associated with greater fluid intake in non-acute hospitalised patients, but both the reasons and the consequences of this relationship, as well as the impact on clinical practice, need to be explored. However, water replacement by oral nutritional support should take advantage of the patients' attitude to assuming a greater fluid intake, limiting at the same time fluid overload during the refeeding phase.
\end{abstract}

Disease-related malnutrition: Nutritional risk at hospital admission: Fluid intake: Food intake: Nutritional Risk Screening 2002

Malnutrition due to both chronic and acute diseases is a widespread, under-recognised and under-treated problem at hospital admission ${ }^{(1)}$. The association between hyporexia/ anorexia, reduced energy intake and disease-related malnutrition has been studied extensively, and most of the underlying pathophysiological mechanisms have been clarified ${ }^{(1,2)}$. Nutritional depletion can also worsen during hospital stay ${ }^{(1,3,4)}$, and clinicians are now aware that nutritional care should be managed continuously during this period. Accordingly, malnutrition screening procedures at referral are now enforced and treatment guidelines have been drawn up, aimed at reducing malnutrition prevalence, the rate of related complications, hospital costs and the length of hospital stay ${ }^{(1,5,6)}$. However, the relationship between nutritional risk and fluid intake has never been investigated. In a healthy subject, body water comprises about $60 \%$ of the total body weight, and it is distributed in two major compartments, extracellular and intracellular. The balance between these compartments and whole body homeostasis strongly depends on ingestion, absorption and retention. During periods of starvation and/or critical illness, water and salt retention usually increases, and both overand under-prescription of fluids can be detrimental. Thus, in the past, researchers focused their attention on the best way to correct fluid and electrolyte imbalances ${ }^{(7,8)}$, but baseline

Abbreviation: PIMAI, Project Iatrogenic Malnutrition in Italy.

The abstract was presented at the 31th ESPEN Congress (Vienna 2009, Austria).

* Corresponding author: Dr Emanuele Cereda, fax +392503 16077, email emanuele.cereda@virgilio.it 
fluid intake has never been investigated either in the critically ill or in a non-intensive setting. However, it can be hypothesised that the pre-existing fluid balance could affect fluid management during the hospital stay.

Taking into consideration this background, the present study was undertaken to evaluate, on admission to hospital, the association between fluid intake and nutritional risk in non-critically ill patients, and to identify factors with potential impact on clinical practice and deserving further investigation.

\section{Methods}

The present study was performed according to the principles of the Declaration of Helsinki, and before any form of assessment, we obtained local Ethics Committee approval. The protocol was approved initially by the Institutional Review Board of the coordinating centre (Bolzano Regional General Hospital), and then by that of the other recruiting centres. We also obtained written informed consent for every patient (the patient itself, relatives or legal guardians). The data analysed in the study were obtained from a larger observational multicentric (thirteen large ( $>400$ beds) multidisciplinary regional hospitals) study, aimed at evaluating the prevalence of malnutrition among hospitalised patients in Italy (Project Iatrogenic Malnutrition in Italy study - PIMAI) ${ }^{(9)}$, and from a personal database of assessments (C. P.) performed at the Hospital of Trento in agreement with the PIMAI study protocol. Thus, the present study is a retrospective analysis of data initially collected for other purposes. All the evaluations were made within $36 \mathrm{~h}$ of admission. Adult patients (age $>18$ years) were recruited from all the wards, except from those of both medical and surgical acute emergencies, according to randomised selection from the daily list of new admissions. A random sample of patients was chosen using the following criteria: one of three patients admitted; no more than three patients/d per hospital; an equal number of males and females; an equal number of subjects aged $<65$ and $\geq 65$ years. Patients whose fluid intake might be altered because of possible disease-related conditions (chronic heart or renal failure, vomiting, diarrhoea, not compensated diabetes, diabetes insipidus and compulsive water drinking) were excluded ${ }^{(10-13)}$. Also, subjects with dementia syndromes, diagnosed according to the International Classification of Disease, 10th Revision $^{(14)}$, those with a moderate to high alcohol intake (set to $\geq 36 \mathrm{~g} / \mathrm{d}$ ) and pregnant women were excluded.

Information was collected on sex, age, educational level, work, speciality of admission, main admission diagnosis, co-morbidities, drugs, overall nutritional risk, determinants of nutritional risk (weight status, recent weight loss and food intake) and fluid intake.

\section{Diagnosis of nutritional risk}

Nutritional risk was diagnosed using the Nutritional Risk Screening 2002 scoring system ${ }^{(5,15)}$. This tool, recommended by the European Society of Parenteral and Enteral Nutrition for nutrition screening, is based on the sum of three different subscores that take into account nutritional status ('Nutritional score'), disease severity ('Disease severity score') and age ('Age score'). The 'Nutritional score' is derived from information on actual body weight as BMI, recent weight loss and period of weight loss ( $\geq 5 \%$ within the last 1,2 or 3 months), and dietary intake during the last week before admission (categorised into quartiles of requirement) as assessed by well-trained dietitians. Energy requirement was calculated as BMR (calculated using the Harris-Benedict equation) corrected by an activity factor or by a stress factor. In estimating dietary intake, we also considered energy from liquid foods. 'Nutritional scoring' is as follows: a score of 3 is assigned to the patient when BMI is $<18.5 \mathrm{~kg} / \mathrm{m}^{2}$, or there has been a weight loss $\geq 5 \%$ in the last month or only $0-25 \%$ of the intake requirement. A score of 2 is given to the patient with $18.5 \mathrm{~kg} / \mathrm{m}^{2} \leq \mathrm{BMI} \leq 20.5 \mathrm{~kg} / \mathrm{m}^{2}$, recent weight loss $\geq 5 \%$ in the last 2 months or an intake of $25-50 \%$ of the requirement. A score of 1 is assigned to the patient when recent weight loss is $\geq 5 \%$ in the last 3 months or the dietary intake is $50-75 \%$ of the requirement.

The 'Disease severity score' categorises the patient according to disease-related metabolic stress $(\approx$ increased requirements); there are three classes: slight (score $=1)$, moderate $($ score $=2)$ or severe $($ score $=3)$. These scores are then added to give a total score to which an additional point is added when age is $\geq 70$ years ('Age score'). Any patient with a total score $\geq 3$ is diagnosed as being 'at nutritional risk'. A more detailed description of this scoring system is provided elsewhere ${ }^{(5,15)}$.

\section{Fluid intake assessment}

Fluid intake assessment was performed by well-trained dietitians, and consisted of the semi-quantitative evaluation of all the major fluid intakes (from sources such as water, juices, sweetened beverages, milk, wine, beer, tea and coffee) the week before admission; this was done by dietary recall and through the use of illustrated atlases. In some cases, additional information was obtained from caregivers. The level of acceptable intake was set at $\geq 5 \mathrm{cups} / \mathrm{d} \quad(>1200 \mathrm{ml} / \mathrm{d}$; 1 cup $=240 \mathrm{ml}$ ). This threshold value was chosen according to commonly accepted statements on fluid balance. In fact, given the normal daily water loss through urine, stools, skin and respiration, the average daily requirement is estimated to be $2500 \mathrm{ml} / \mathrm{d}$, with approximately $1000-1500 \mathrm{ml}$ coming from beverages and the remaining amount coming from substrate oxidation and foods ${ }^{(16,17)}$.

\section{Possible risk factors for altered fluid intake}

Apart from age, the presence of malignancies (cancer), diabetes and the number of co-morbidities were investigated as possible independent risk factors ${ }^{(13,18,19)}$. The diagnosis of 'being at nutritional risk', the adequacy of dietary intake in the previous week (assessed as quartiles of requirement: $<25 \%, 25-<50 \%, 50-<75 \%$ and $\geq 75 \%$ ) and recent body weight loss ( $\geq 5 \%$ in 1,2 or 3 months) were also considered. Finally, the use of blood pressure-lowering medications and diuretics, as well as the daily number of prescribed drugs (drugs/d; including antihypertensive medications and diuretics), were taken into account as potential determinants of fluid intake ${ }^{(17)}$. Accordingly, the patients were stratified as follows: $0-2,3-5$ and $\geq 6 \mathrm{drugs} / \mathrm{d}$. 
Table 1. Study sample distribution according to setting, speciality, nutritional risk and low fluid intake $(<5$ cups/d)

\begin{tabular}{lrrrr}
\hline & \multicolumn{2}{c}{ Total } & & \\
\cline { 2 - 3 } Setting and speciality & $n$ & $\%$ & At-risk (\%) & Low fluid intake (\%) \\
\hline Medical & & & & \\
$\quad$ General medicine & 128 & $22 \cdot 9$ & $60 \cdot 9$ & $44 \cdot 5$ \\
Cardiology & 14 & $2 \cdot 3$ & $50 \cdot 0$ & $42 \cdot 9$ \\
Endocrinology/metabolism & 8 & $1 \cdot 4$ & $50 \cdot 0$ & $25 \cdot 0$ \\
Gastroenterology & 19 & $3 \cdot 4$ & $78 \cdot 9$ & $26 \cdot 3$ \\
Geriatrics & 26 & $4 \cdot 7$ & $69 \cdot 6$ & $88 \cdot 5$ \\
Haematoloy/oncology & 37 & $6 \cdot 6$ & $78 \cdot 4$ & $51 \cdot 4$ \\
Immunology/infectivology & 23 & $4 \cdot 1$ & $69 \cdot 6$ & $47 \cdot 8$ \\
Neurology & 33 & $5 \cdot 9$ & $39 \cdot 4$ & $60 \cdot 6$ \\
Nephrology & 10 & $1 \cdot 8$ & $70 \cdot 0$ & $60 \cdot 0$ \\
Pneumology & 19 & $3 \cdot 4$ & $73 \cdot 4$ & $42 \cdot 1$ \\
Psychiatry & 15 & $2 \cdot 7$ & $26 \cdot 7$ & $40 \cdot 0$ \\
Rheumatology/dermatology & 9 & $1 \cdot 6$ & $33 \cdot 3$ & $55 \cdot 5$ \\
Others & 22 & $3 \cdot 9$ & $77 \cdot 3$ & $40 \cdot 9$ \\
Surgical & & & & \\
General/abdominal surgery & 74 & $13 \cdot 2$ & $62 \cdot 2$ & $32 \cdot 4$ \\
Cardiothoracic surgery & 21 & $3 \cdot 8$ & $47 \cdot 6$ & $33 \cdot 3$ \\
Maxillary/plastic/vascular surgery & 11 & $2 \cdot 0$ & $54 \cdot 5$ & $45 \cdot 4$ \\
Neurosurgery & 21 & $3 \cdot 8$ & $33 \cdot 3$ & $42 \cdot 9$ \\
Gynaecological surgery & 13 & $2 \cdot 3$ & $53 \cdot 8$ & $46 \cdot 2$ \\
Orthopaedic/traumatology & 27 & $4 \cdot 8$ & $37 \cdot 0$ & $44 \cdot 4$ \\
Otorhinolaringology & 16 & $2 \cdot 9$ & $43 \cdot 7$ & $50 \cdot 0$ \\
Urology & 13 & $2 \cdot 3$ & $46 \cdot 2$ & $76 \cdot 9$ \\
\hline & & & & \\
\hline & & & & \\
\hline
\end{tabular}

\section{Statistical analysis}

All statistical analyses were performed using the SPSS $16 \cdot 0$ statistical package (SPSS for Windows; SPSS Inc., Chicago, IL, USA).

The normal distribution of the variables was examined using the Kolmogorov-Smirnov test. Continuous variables of the two groups were compared using unpaired Student's $t$ test (normal distribution) or the Mann-Whitney $U$ test (not normal distribution), whichever was appropriate. However, multiple-group comparison was performed using ANOVA or the Kruskal-Wallis test. Frequencies were compared using the $\chi^{2}$ test. Finally, we investigated the independent determinants of reduced fluid intake ( $<5 \mathrm{cups} / \mathrm{d}$; dependent variable) using logistic regression models, and OR with $95 \%$ CI were calculated. First, we ran univariate analyses adjusted for sex, height, educational level, work, admission speciality and recruiting centre. All the correlates with a $P<0 \cdot 10$ by these models were then analysed using multivariate analysis, basing the analysis on the crude model and making additional adjustments for diabetes and the use of diuretics and medication for lowering blood pressure. In all the models, included independent variables were treated categorically. Statistical significance for type I error was established by $P<0 \cdot 05$.

\section{Results}

From the initial population fulfilling the inclusion criteria ( $n$ 2136), we excluded patients due to the following reasons: $n 252(11.8 \%)$, refusal to participate or terminal illness; $n 301$ (14.1\%), unavailability of complete data to perform risk assessment using the Nutritional Risk Screening 2002 tool; $n 854$ (40\%), lack of fluid intake assessment (not performed since the beginning of the recruitment phase because it was not included in the initial study protocol); $n 170(8.0 \%)$, fluid intake potentially altered because of disease-related conditions.

Final analysis included a study sample of 559 subjects. Patients were recruited from all the regular wards (Table 1), but most of them were from internal medicine $(22.9 \%)$ and general surgery $(13.2 \%)$. Overall prevalence of nutritional risk was $57.2 \%$ ( $n$ 320), with significantly higher rates in the medical setting than in the surgical one $(61.2 v .50 .5 \%)$ (Table 2). The prevalence of patients 'at risk' and of those reporting low fluid intake $(<5 \mathrm{cups} / \mathrm{d})$ was markedly heterogeneous among the investigated specialities, and it ranged from $26.7 \%$ (psychiatry) to $78.9 \%$ (gastroenterology) for nutritional risk, and from $25.0 \%$ (endocrinology) to $88.5 \%$ (geriatrics) for fluid intake. A weight loss $\geq 5 \%$ and an energy intake $<75 \%$ of the estimated requirement were recorded in 62.3 and $36.4 \%$ of the cases, respectively. Both these features were reported by $24.2 \%$ of the patients. 'At-risk' patients showed lower BMI, albumin and prealbumin serum levels, and a higher number of prescribed daily drugs taken, and were more likely to suffer malignancies and report a higher fluid intake (Table 2).

Therefore, the patients were divided into four groups according to fluid intake and nutritional risk (Table 3). The differences in BMI, albumin, prealbumin and lymphocytes between 'at-risk' and 'not at-risk' patients appeared to be independent of fluid intake. Patients 'at risk' and characterised by poor fluid intake were significantly older than those in the other groups. We also found a significant association with the entity of weight loss, food intake and the number of medications taken. It was noted that the 'not at-risk' patients with poor fluid intake and the patients 'at risk' with acceptable fluid intake, respectively, took the lowest and highest average number of drugs. 
Table 2. Features of the population according to nutritional risk*

(Mean values and standard deviations)

\begin{tabular}{|c|c|c|c|c|c|c|c|c|c|c|}
\hline & \multicolumn{3}{|c|}{ Overall ( $n$ 559) } & \multicolumn{3}{|c|}{ At-risk ( $n$ 320) } & \multicolumn{3}{|c|}{ Not at-risk ( $n$ 239) } & \multirow[b]{2}{*}{$P \ddagger$} \\
\hline & $\% \dagger$ & Mean & SD & $\% \dagger$ & Mean & SD & $\% \dagger$ & Mean & SD & \\
\hline Ward (surgical:medical) & $206: 353$ & & & $104: 216$ & & & $102: 137$ & & & $<0.02$ \\
\hline $\operatorname{Sex}(M: F)$ & $280: 279$ & & & $155: 165$ & & & $125: 114$ & & & 0.37 \\
\hline Age (years) & & $62 \cdot 4$ & $18 \cdot 8$ & & $63 \cdot 2$ & $18 \cdot 8$ & & $61 \cdot 3$ & $18 \cdot 9$ & 0.25 \\
\hline BMI $\left(\mathrm{kg} / \mathrm{m}^{2}\right)$ & & 24.4 & $5 \cdot 4$ & & $22 \cdot 5$ & 4.5 & & $27 \cdot 1$ & 5.4 & $<0.001$ \\
\hline Albumin $(g / l)$ & & $36 \cdot 9$ & $7 \cdot 0$ & & $35 \cdot 2$ & 7.9 & & 39.4 & $4 \cdot 1$ & $<0.001$ \\
\hline Prealbumin (mg/l) & & 202 & 94 & & 173 & 86 & & 259 & 83 & $<0.001$ \\
\hline Lymphocytes $\left(/ \mathrm{mm}^{3}\right)$ & & 1670 & 865 & & 1414 & 757 & & 2044 & 880 & $<0.001$ \\
\hline \multicolumn{11}{|l|}{ WL } \\
\hline$\geq 5 \%$ within 1 month & $21 \cdot 3$ & & & $37 \cdot 2$ & & & 0 & & & $<0.001$ \\
\hline$\geq 5 \%$ within 2 months & $28 \cdot 8$ & & & $30 \cdot 4$ & & & $26 \cdot 8$ & & & \\
\hline$\geq 5 \%$ within 3 months & $12 \cdot 2$ & & & $9 \cdot 1$ & & & $16 \cdot 4$ & & & \\
\hline \multicolumn{11}{|l|}{ Food intake } \\
\hline$<25 \%$ & $2 \cdot 7$ & & & 4.7 & & & 0 & & & $<0.001$ \\
\hline $25-50 \%$ & $8 \cdot 1$ & & & 11.9 & & & $3 \cdot 0$ & & & \\
\hline $50-75 \%$ & $25 \cdot 6$ & & & 32.5 & & & $16 \cdot 3$ & & & \\
\hline Co-morbidities $(n)$ & & $1 \cdot 3$ & 1.4 & & 1.3 & 1.5 & & $1 \cdot 3$ & 1.4 & 0.79 \\
\hline Malignancy & $20 \cdot 7$ & & & $25 \cdot 9$ & & & $13 \cdot 8$ & & & $<0.001$ \\
\hline Diabetes & $13 \cdot 9$ & & & $11 \cdot 6$ & & & $17 \cdot 1$ & & & 0.06 \\
\hline Drugs $(n)$ & & $3 \cdot 1$ & $2 \cdot 6$ & & $3 \cdot 3$ & $2 \cdot 7$ & & $2 \cdot 8$ & 2.5 & 0.04 \\
\hline Diuretics & $14 \cdot 5$ & & & $16 \cdot 6$ & & & $11 \cdot 7$ & & & 0.55 \\
\hline Antihypertensives & $17 \cdot 0$ & & & $17 \cdot 8$ & & & $15 \cdot 9$ & & & 0.11 \\
\hline \multicolumn{11}{|l|}{ Fluid } \\
\hline$<5$ cups $/ \mathrm{d}$ & $46 \cdot 2$ & & & $35 \cdot 0$ & & & $53 \cdot 1$ & & & $<0.005$ \\
\hline$\geq 5 \mathrm{cups} / \mathrm{d}$ & 53.8 & & & $65 \cdot 0$ & & & $46 \cdot 9$ & & & \\
\hline
\end{tabular}

$\mathrm{M}$, male; $\mathrm{F}$, female; WL, weight loss before hospital admission.

${ }^{*}$ Data are presented as prevalence/frequency as indicated.

†Percentages refer to the whole group (within a single column).

$\ddagger$ According to unpaired $t$ test or Mann-Whitney $U$ test and $\chi^{2}$ test, where appropriate.

Finally, we investigated the independent predictors of low fluid intake $(<5$ cups/d; dependent variable) at hospital admission using multivariate logistic regression models (Table 4). Multiple-adjusted univariate analyses revealed that patients aged $\geq 65$ years, presenting with reduced energy intake and a lower number of drugs taken, were more likely to have a lower fluid intake. Moreover, higher fluid intake was detected in patients with decreasing BMI and those 'at nutritional risk'. The multivariate model also confirmed similar associations, the exception being that with weight status.

\section{Discussion}

Disease-related malnutrition has been proven to be frequently associated with reduced food/energy intake, and this, in turn, significantly contributes to body mass wasting and impairment of functional status ${ }^{(1,2,4)}$. Even dehydration of as little as $2 \%$ leading to loss of body weight can result in impaired physiological responses and functional change ${ }^{(8,17)}$. However, the failure to prescribe adequate fluids also, resulting in overload, might have consequences in terms of complications or recovery rate after surgery or a critical illness ${ }^{(7,8)}$. These pathological conditions, as well as many other diseases, are responsible for higher nutritional risk ${ }^{(1,5)}$, but the relationship between nutritional status or risk and oral fluid consumption on admission to hospital has been never explored.

In the present study, we observed for the first time that non-critically ill patients at nutritional risk are more likely to present with an acceptable fluid intake. We also detected an independent association between fluid intake and age, food intake and multiple pharmacological treatment.

It has been well demonstrated that levels of thirst reduce progressively during ageing due to a decrease in the sensitivity of volume- and osmoreceptors ${ }^{(13,20)}$. Along with this, a role for mental (e.g. decline of cognitive function) and physical dependency, as well as for behavioural changes, should be recognised $^{(13)}$.

We observed a direct relationship between the energy and fluid intakes. It is reasonable to argue that subjects eating to a normal extent drink more to accompany food. Food per se is an important source of water, but some energy can also be introduced in liquid form (wine, milk, beverages, etc.).

Also, the positive association between polypharmacy and fluid intake may be simple to explain as the ingestion of medication frequently takes place during meals, and the patients usually have a cup of water or other liquids everytime they need to swallow a pill.

However, some liquid preloads are able to reduce appetite if they provide energy, particularly in the elderly ${ }^{(21)}$, and drugs are known to negatively affect energy intake ${ }^{(1,21)}$. Malnourished patients are more likely to be older, and frequently present reduced energy intake and multiple drug prescriptions, which are interacting factors that result in detrimental consequences on nutritional status ${ }^{(1,5,22,23)}$. However, the association between nutritional risk and fluid intake was found to be independent. Thus, the mechanisms beyond this relationship are not clear, and some speculations would be in order.

When disease is present, particularly acute injury or critical illness, the inflammatory condition induces a generalised 
Table 3. Features of the population according to fluid intake and nutritional risk (Nutritional Risk Screening 2002 score $\geq 3$ ) $\dagger$ (Mean values and standard deviations)

\begin{tabular}{|c|c|c|c|c|c|c|c|c|c|c|c|c|c|}
\hline & \multicolumn{6}{|c|}{$<5$ (cups/d) } & \multicolumn{6}{|c|}{$\geq 5$ (cups/d) } & \multirow[b]{3}{*}{$P \S$} \\
\hline & \multicolumn{3}{|c|}{ At-risk ( $n$ 131) } & \multicolumn{3}{|c|}{ Not at-risk ( $n 127)$} & \multicolumn{3}{|c|}{ At-risk ( $n$ 189) } & \multicolumn{3}{|c|}{ Not at-risk ( $n 112)$} & \\
\hline & $\% \ddagger$ & Mean & SD & $\% \ddagger$ & Mean & SD & $\% \ddagger$ & Mean & SD & $\% \ddagger$ & Mean & SD & \\
\hline Ward (surgical:medical) & $38: 93$ & & & $47: 80$ & & & $66: 123$ & & & $55: 57$ & & & 0.02 \\
\hline $\operatorname{Sex}(M: F)$ & $64: 67$ & & & $67: 60$ & & & $91: 98$ & & & $58: 54$ & & & 0.84 \\
\hline Age (years) & & $67 \cdot 3^{*}$ & $17 \cdot 1$ & & 63.4 & 18.5 & & $60 \cdot 4$ & $19 \cdot 4$ & & $59 \cdot 0$ & $19 \cdot 2$ & $<0.002$ \\
\hline BMI $\left(\mathrm{kg} / \mathrm{m}^{2}\right)$ & & $22 \cdot 7^{*}$ & 4.3 & & 28.0 & 5.5 & & $22 \cdot 4^{*}$ & 4.6 & & $26 \cdot 6$ & $5 \cdot 2$ & $<0.001$ \\
\hline Albumin $(g / /)$ & & $34.1^{*}$ & 8.0 & & $39 \cdot 4$ & 4.0 & & $36 \cdot 1^{\star}$ & $7 \cdot 7$ & & 39.5 & $4 \cdot 3$ & $<0.001$ \\
\hline Prealbumin (mg/l) & & $163^{\star}$ & 74 & & 279 & 83 & & $179^{*}$ & 92 & & 244 & 81 & $<0.001$ \\
\hline Lymphocytes $\left(/ \mathrm{mm}^{3}\right)$ & & $1450^{*}$ & 823 & & 2012 & 899 & & $1391^{*}$ & 712 & & 2081 & 861 & $<0.001$ \\
\hline \multicolumn{14}{|l|}{ WL } \\
\hline$\geq 5 \%$ within 1 month & 37.4 & & & 0 & & & 37.0 & & & 0 & & & $<0.001$ \\
\hline$\geq 5 \%$ within 2 months & $34 \cdot 3$ & & & $26 \cdot 8$ & & & 27.5 & & & $26 \cdot 8$ & & & \\
\hline$\geq 5 \%$ within 3 months & $11 \cdot 4$ & & & $21 \cdot 2$ & & & $7 \cdot 4$ & & & $10 \cdot 7$ & & & \\
\hline \multicolumn{14}{|l|}{ Food intake } \\
\hline$<25 \%$ & 9.9 & & & 0 & & & $1 \cdot 1$ & & & 0 & & & $<0.001$ \\
\hline $25-50 \%$ & 24.4 & & & 4.7 & & & $3 \cdot 2$ & & & 0.9 & & & \\
\hline $50-75 \%$ & 38.9 & & & $24 \cdot 4$ & & & $28 \cdot 0$ & & & $7 \cdot 1$ & & & \\
\hline Co-morbidities $(n)$ & & 1.5 & 1.5 & & 1.3 & 1.3 & & 1.2 & 1.4 & & 1.2 & 1.5 & 0.13 \\
\hline Malignancy & $27 \cdot 5$ & & & $15 \cdot 7$ & & & $24 \cdot 9$ & & & 11.6 & & & 0.09 \\
\hline Diabetes & 9.9 & & & $18 \cdot 9$ & & & $12 \cdot 7$ & & & $15 \cdot 2$ & & & 0.19 \\
\hline Drugs $(n)$ & & 3.1 & $2 \cdot 7$ & & $2 \cdot 4^{*}$ & $2 \cdot 3$ & & $3.5^{\star}$ & $2 \cdot 7$ & & 3.3 & $2 \cdot 8$ & 0.01 \\
\hline Diuretics & 13.7 & & & $15 \cdot 0$ & & & $18 \cdot 5$ & & & 8.0 & & & $0 \cdot 10$ \\
\hline Antihypertensives & $19 \cdot 1$ & & & $15 \cdot 0$ & & & $16 \cdot 9$ & & & 17.0 & & & 0.86 \\
\hline
\end{tabular}

$M$, male; $F$, female; WL, weight loss before hospital admission.

${ }^{*}$ Mean values were significantly different, post hoc comparison by Scheffe's test $(P<0.05)$.

† Data are presented as prevalence/frequency as indicated.

$\ddagger$ Percentages refer to the whole group.

$\S P$ values according to ANOVA or Kruskal-Wallis test and $\chi^{2}$ test, where appropriate. 
Table 4. Logistic regression models of independent risk factors for low fluid intake ( $<5$ cups/d) (Odds ratios and $95 \%$ confidence intervals)

\begin{tabular}{|c|c|c|c|c|c|c|}
\hline \multirow[b]{2}{*}{ Risk factor } & \multicolumn{3}{|c|}{ Univariate model ${ }^{*}$} & \multicolumn{3}{|c|}{ Multivariate model $\dagger$} \\
\hline & OR & $95 \% \mathrm{Cl}$ & $P$ & OR & $95 \% \mathrm{Cl}$ & $P$ \\
\hline Setting (surgical $v$. medical) & 0.81 & $0.52,1.25$ & 0.34 & - & & \\
\hline Age $\geq 65$ years & $1 \cdot 60$ & $1.04,2.45$ & 0.03 & 1.88 & $1.03,3.43$ & 0.04 \\
\hline Weight status $\ddagger$ & $1 \cdot 27$ & $1.02,1.58$ & 0.03 & $1 \cdot 26$ & $0.92,1.74$ & 0.15 \\
\hline Weight loss§ & 0.94 & $0.81,1.09$ & 0.39 & - & & \\
\hline Food intake\| & 0.54 & $0.39,0.75$ & $<0.001$ & 0.37 & $0.25,0.55$ & $<0.001$ \\
\hline Co-morbidities & $1 \cdot 10$ & $0.97,1.25$ & 0.13 & - & & \\
\hline Malignancy & $1 \cdot 18$ & $0.77,1.82$ & 0.45 & - & & \\
\hline Drugsף & 0.62 & $0.48,0.79$ & $<0.001$ & 0.63 & $0.44,0.90$ & 0.02 \\
\hline 'At nutritional risk'”* & 0.58 & $0.41,0.81$ & $<0.002$ & 0.64 & $0.43,0.95$ & 0.03 \\
\hline
\end{tabular}

* Adjusted for sex, height, educational level, work, recruiting centre and admission speciality.

$\dagger$ Further adjusted for diabetes, use of diuretics and blood pressure-lowering medications.

$\ddagger$ Entered as categorical variable: underweight, 0; normal weight, 1; overweight, 2; obesity, 3.

\& Entered as categorical variable: $\geq 5 \%$ in 1 month, $0 ; \geq 5 \%$ in 2 months, $1 ; \geq 5 \%$ in 3 months, 2 .

|| Entered as categorical variable: $<25 \%$ requirement, $0 ; 25-<50 \%$ requirement, $1 ; 50-<75 \%$ requirement, $2 ; \geq 75 \%$ requirement, 3.

ๆ Entered as categorical variable: 0-2 drugs/d, 0; 3-5 drugs/d, 1; 6 or more drugs/d, 2.

${ }^{\star *}$ As defined by Nutritional Risk Screening 2002 score $\geq 3$.

increase in capillary permeability with a leakage of albumin, and so of fluids, to the extravascular space s,8,24-26) $^{(7 l b u m i n}$ levels fall also for an impairment in protein turnover ${ }^{(25)}$. Moreover, the alteration electrolyte fluxes across cellular membrane due to ATP depletion should be taken into consideration. Accordingly, low plasma flow and electrolyte alterations can lead to the activation of volume- and osmoreceptors, resulting in an increase in water and $\mathrm{Na}$ retention and in the sense of thirst ${ }^{(7,8,20)}$.

The aforementioned pathophysiological mechanisms apparently refer to the two main types of malnutrition, kwashiorkor-like and marasmatic, and they could be useful to distinguish between them. Indeed, it has been demonstrated that the two conditions frequently coexist, and that both contribute to nutritional risk $^{(1)}$. Perhaps, the availability of data concerning inflammatory markers (e.g. C-reactive protein) would better sustain this hypothesis, and an evaluation of body fluid compartments (intracellular and extracellular) and natraemia would help explain this relationship. Nonetheless, part of the independent association found with nutritional risk could be explained by the tool used for risk assessment. The Nutritional Risk Screening 2002 tool was developed and validated in the acute care setting, and focusing much more on the effect of a disease, it seems to better reflect the inflammatory background associated with the pathological process leading to hospitalisation ${ }^{(15)}$.

We do not know the consequences of the present results on outcome and clinical practice. Indeed, fluid intakes are different from fluid status. Water and salt retention is usually increased in starved subjects, and both over- and underprescription of fluids can be detrimental. Accordingly, a cautious correction of even modest deficits or excesses of fluid and electrolyte imbalances is recommended to avoid excess fluid retention and to optimise physiological functions, particularly in those patients suffering from critical illness or acute injury (e.g. burn or brain injury) or who have undergone surgical procedures ${ }^{(7,8)}$. In our study, the evaluation was performed at admission, thus before surgery, and most critically ill patients were excluded.
It is not clear if this attitude to a better fluid intake affects patient prognosis, and we are unable to set a threshold fluid intake level, over or under which the rates of complications might change significantly. It should be recognised that no real estimation of fluid balance was performed, as we did not consider output, loss or water coming from solid foods.

We cannot suggest that 'at-risk' patients be advised to change their behaviour. Indeed, in the initial phase of refeeding, water replacement by oral energy-dense formula could be advantageous for 'at-risk' patients, as the increase in both fluid and energy needs by this route seems feasible and effective ${ }^{(27)}$, and this practice would also be in agreement with the need to avoid fluid overload during the refeeding process ${ }^{(26,28)}$.

The study has limitations other than those presented earlier. Indeed, the method used for assessing fluid intake (recall) is useful in population studies, and similarly to food intake, it is well applicable in this type of setting. The major disadvantage is its heavy reliance on memory, as well as that it also overlooks day-to-day food variation. Unfortunately, the use of quantitative tools of assessment, such as diaries, cannot be considered in such a study design. Limits should also be recognised in the lack of distinction between the different fluid sources (energetic and non-energetic) and in the threshold value chosen to define an acceptable intake. The one reported is suggested in the literature, but it refers to the real fluid balance.

The exclusion of some patient categories is also recognised as a limit, as well as the type of study design (retrospective rather than prospective). Accordingly, our findings should be interpreted cautiously, and they could not be generalised to the whole population of patients at risk of malnutrition at hospital referral. Some of the excluded pathological conditions are related to both nutritional risk and alteration of fluid intake (e.g. chronic heart or renal failure, persistent vomiting or diarrhoea, dementia and total dependence in the activities of daily living), but the inclusion would have been confounding. However, an extensive evaluation of cognitive function was not performed, and only patients with a reported diagnosis of dementia were excluded. 
In conclusion, non-acute patients at risk of malnutrition or with overt malnutrition at hospital admission frequently present with better fluid intake. In critically ill patients, fluid overload must be avoided. Thus, our observations in the non-critically ill patients suggest that the evaluation of fluid balance at hospital admission is of potential interest and deserves investigation. It cannot be excluded that a better focus on this issue might be helpful in improving the nutritional management of hospitalised patients.

\section{Acknowledgements}

The present study was partially supported by grants of Nutricia (Milan, Italy). All authors certify that there are no affiliations with or involvement in any organisation or entity with a direct financial interest in the subject matter or materials discussed in the manuscript. All the authors significantly contributed to the work, read and approved the final manuscript. E. C. designed the study, analysed data and wrote the manuscript. L. L., A. D'. A., M. G. G., N. C. B. and M. A. F. designed the PIMAI study. C. P. collected part of the data and contributed to their interpretation and manuscript drafting. All the authors belonging to the PIMAI group contributed to data collection and interpretation. We are particularly grateful to all the contributing centres (the PIMAI group) and the relative personnel (nutritional scientists, dietitians and nurses) involved in data collection:

1. Dietetic and Clinical Nutrition Unit, Regional General Hospital Bolzano: Lucchin L, Lando L, Borgo S, Saffiotti GL.

2. Dietetic and Clinical Nutrition Unit, 'Niguarda-Ca Granda' Hospital, Milano: Gentile MG, Rodeschini E, Sandri LG.

3. Dietetic and Clinical Nutrition Unit, 'Maggiore della Carità' Hospital, Novara: D’Andrea F, Brugnani M, Barbero B, Passera S.

4. Dietetic and Clinical Nutrition Unit, University Hospital, Padova: Caregaro L, Nardi MT.

5. Dietetic and Clinical Nutrition Unit, 'S.Martino' Hospital, Genova: Sukkar GS, Ferrari C.

6. Dietetic and Clinical Nutrition Unit, 'Le Scotte' University Hospital, Siena: Mattei R, Grosso A, Francalanzi C, Cardinali F, Borsi E.

7. Dietetic and Clinical Nutrition Unit, 'Casa Sollievo della Sofferenza' Hospital, S.Giovanni Rotondo, Foggia: Orban A, Cianti L.

8. Endocrinology, Diabetology and Clinical Nutrition Unit, 'S.Sebastiano' Hospital, Caserta: Prilli M, Capriello R, Sorrentino S, Pennino MR.

9. Dietetic and Clinical Nutrition Unit, 'Canizzarro' Hospital, Catania: Leonardi F, Bellino AME, Massimino EA.

10. Dietetic and Clinical Nutrition Unit, 'Umberto I' Hospital, Ancona: Nicolai A, Petrelli M, Taus M, Busni D, Borri MG, Vitrini S.

11. Dietetic and Clinical Nutrition Unit, 'A.Cardarelli' Hospital, Campobasso: Pastò S, Di Biase P, D’Onofrio R, Di Brino AM, Celi C, Mastronuzzi V.

12. Department of Internal Medicine, University 'La Sapienza', Roma: Muscaritoli M, Preziosa I, Canali A.
13. Dietetic and Clinical Nutrition Unit, 'S.Maria' Hospital, Terni: Fatati G, Mirri E, Palazzi M, Vendetti AL, Sette S, Panetta V.

14. National Institute for Research on Food and Nutrition (INRAN), Rome.

\section{References}

1. Norman K, Pichard C, Lochs H, et al. (2008) Prognostic impact of disease-related malnutrition. Clin Nutr 27, 5-15.

2. Evans WJ, Morley JE, Argilés J, et al. (2008) Cachexia: a new definition. Clin Nutr 27, 793-799.

3. McWhirter JP \& Pennington CR (1994) Incidence and recognition of malnutrition in hospital. BMJ 308, 945-948.

4. Incalzi RA, Gemma A, Capparella O, et al. (1996) Energy intake and in-hospital starvation. A clinically relevant relationship. Arch Intern Med 156, 425-429.

5. Kondrup J, Allison SP, Elia M, et al. (2002) ESPEN guidelines for nutrition screening 2002. Clin Nutr 22, 415-421.

6. Lochs H, Allison SP, Meier R, et al. (2006) Introductory to the ESPEN Guidelines on enteral nutrition: terminology, definitions and general topics. Clin Nutr 25, 180-186.

7. Lobo DN (2004) Fluid, electrolytes and nutrition: physiological and clinical aspects. Proc Nutr Soc 63, 453-466.

8. Allison S (2004) Fluid, electrolytes and nutrition. Clin Med 4, 573-578.

9. Lucchin L, D’Amicis A, Gentile MG, et al. (2009) An Italian investigation on nutritional risk at hospital admission: the PIMAI (Project: Iatrogenic MAlnutrition in Italy) study. Eur $J$ Clin Nutr Metab 4, e199-e202.

10. Sung JM, Kuo SC, Guo HR, et al. (2006) The role of oral dryness in interdialytic weight gain by diabetic and non-diabetic haemodialysis patients. Nephrol Dial Transplant 21, $2521-2528$

11. Holst M, Strömberg A, Lindholm M, et al. (2008) Description of self-reported fluid intake and its effects on body weight, symptoms, quality of life and physical capacity in patients with stable chronic heart failure. J Clin Nurs 17, 2318-2326.

12. Manz F (2007) Hydration and disease. J Am Coll Nutr 26, Suppl. 5, 535S-541S.

13. Allison SP \& Lobo DN (2004) Fluid and electrolytes in the elderly. Curr Opin Clin Nutr Metab Care 7, 27-33.

14. World Health Organization (2007) International Classification of Disease, 10th Revision. http://www.who.int/classifications/ apps/icd/icd10online/

15. Kondrup J, Rasmussen HH, Hamberg O, et al. (2003) Nutritional risk screening (NRS 2002): a new method based on an analysis of controlled clinical trials. Clin Nutr 22, 321-336.

16. Istituto Nazionale di Ricerca per gli Alimenti e Nutrizione [INRAN] - Ministero delle Politiche Agricole, Alimentari e Forestali (2003) Italian guidelines for a healthy nutrition. http://www.inran.it/servizi_cittadino/stare_bene/guida_corretta_ alimentazione/Linee\%20Guida.pdf

17. Kleiner SM (1999) Water: an essential but overlooked nutrient. J Am Diet Assoc 99, 200-206.

18. Sarhill N, Mahmoud FA, Christie R, et al. (2003) Assessment of nutritional status and fluid deficits in advanced cancer. Am $J$ Hosp Palliat Care 20, 465-473.

19. DeFronzo RA (1981) The effect of insulin on renal sodium metabolism. A review with clinical implications. Diabetologia 21, 165-171.

20. Farrell MJ, Zamarripa F, Shade R, et al. (2008) Effect of aging on regional cerebral blood flow responses associated with osmotic thirst and its satiation by water drinking: a PET study. Proc Natl Acad Sci U S A 105, 382-387. 
21. Sturm K, Parker B, Wishart J, et al. (2004) Energy intake and appetite are related to antral area in healthy young and older subjects. Am J Clin Nutr 80, 656-667.

22. Omran ML \& Morley JE (2000) Assessment of protein energy malnutrition in older persons, part I: history, examination, body composition, and screening tools. Nutrition 16, 50-63.

23. Pirlich M, Schütz T, Norman K, et al. (2006) The German hospital malnutrition study. Clin Nutr 25, 563-572.

24. Anderson J, Lawes S, Lobo D, et al. (2002) Sequential changes in serum albumin c-reactive protein and transcapillary escape rate of albumin in patients undergoing major abdominal surgery. Clin Nutr 21, Suppl. 1, 29.
25. Ballmer PE (2001) Causes and mechanisms of hypoalbuminaemia. Clin Nutr 20, 271-273.

26. Stanga Z, Brunner A, Leuenberger M, et al. (2008) Nutrition in clinical practice - the refeeding syndrome: illustrative cases and guidelines for prevention and treatment. Eur J Clin Nutr 62, 687-694.

27. Eneroth M, Olsson UB \& Thorngren KG (2005) Insufficient fluid and energy intake in hospitalised patients with hip fracture. A prospective randomised study of 80 patients. Clin Nutr 24, 297-303.

28. Mehanna HM, Moledina J \& Travis J (2008) Refeeding syndrome: what it is, and how to prevent and treat it. $B M J$ 336, 1495-1498. 